\title{
Adaptive Shared Control of a Brain-Actuated Simulated Wheelchair
}

\author{
Johan Philips, José del R. Millán, Gerolf Vanacker, Eileen Lew, Ferran Galán, \\ Pierre W. Ferrez, Hendrik Van Brussel and Marnix Nuttin
}

\begin{abstract}
The use of shared control techniques has a profound impact on the performance of a robotic assistant controlled by human brain signals. However, this shared control usually provides assistance to the user in a constant and identical manner each time. Creating an adaptive level of assistance, thereby complementing the user's capabilities at any moment, would be more appropriate. The better the user can do by himself, the less assistance he receives from the shared control system; and vice versa. In order to do this, we need to be able to detect when and in what way the user needs assistance. An appropriate assisting behaviour would then be activated for the time the user requires help, thereby adapting the level of assistance to the specific situation. This paper presents such a system, helping a brain-computer interface (BCI) subject perform goal-directed navigation of a simulated wheelchair in an adaptive manner. Whenever the subject has more difficulties in driving the wheelchair, more assistance will be given. Experimental results of two subjects show that this adaptive shared control increases the task performance. Also, it shows that a subject with a lower BCI performance has more need for extra assistance in difficult situations, such as manoeuvring in a narrow corridor.
\end{abstract}

\section{INTRODUCTION}

Nowadays, most people who are paraplegic or have another physical impairment at the lower limbs can be provided with a fair amount of mobility and independence through the use of an ordinary wheelchair, either manual or electrical. However, the use of such a mechanical device cannot provide aid to all people. Imagine someone with an uncontrollable tremor in his hand or arm trying his best to make a safe passage through a narrow corridor using an electrical wheelchair. For those people an intelligent controller inside the wheelchair together with range sensors, detecting nearby obstacles, could solve many of problems [1]. Theoretically, the person could then switch on this controller and it would autonomously drive the wheelchair through the corridor, while avoiding all obstacles. Though this might be appropriate for other applications, the wheelchair user loses the feeling of continuous control. This loss of independence is undesirable and therefore, shared control between the user and the controller is more suitable in these cases. There have been promising results in this field recently, where the shared control system estimates the user's intention and provides aid accordingly [2], [3].

Nevertheless, there are still people who cannot directly benefit from this technology due to their severe physical impairment. Tetraplegics, whose paralysis prevents them

This work is supported by the European IST Programme FET Project FP6-003758. This paper only reflects the authors' views and funding agencies are not liable for any use that may be made of the information contained herein.

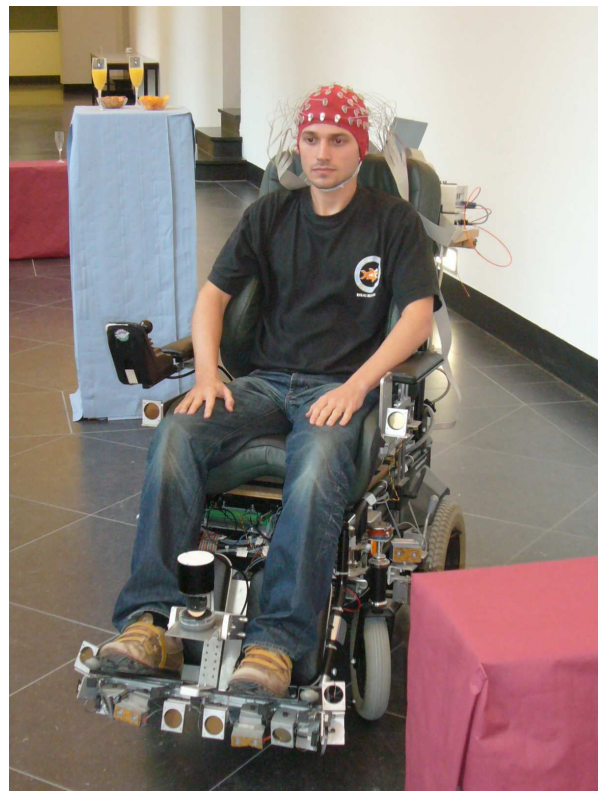

Fig. 1. The subject wearing an electroencephalogram (EEG) sensor cap is manoeuvring the robot wheelchair Sharioto through a natural indoor environment. The electrodes on the sensor cap are connected to the BCI system through an analogue to digital converter and amplifier.

from using an ordinary joystick of an electrical wheelchair by hand or patients with locked-in syndrome, require other technologies. Examples are the chin joysticks, which can be mounted on the wheelchair, or the eye and gaze tracking techniques [4]. Another promising technology is braincomputer interface (BCI) control of a mechanical device.

Although the idea of mentally controlling a common apparatus is certainly appealing, the complexity of an everyday environment increases the difficulty to design a robust system, capable of coping with such a complexity. Nevertheless, more recently, there seems to be an increase in the research done on non-invasive brain-computer interfaces. The key motivation of this research is to provide aid for people whose impairment is so severe that current solutions are not suitable. BCI control could offer them a way to improve their communication, increase their mobility and independence again. Typical applications would be controlling an internet browser or word processor [5], [6], [7], prostheses and mobility aids, such as a wheelchair [8]. 
More recently, in the MAIA project ${ }^{1}$ the asynchronous IDIAP BCI [9] has been integrated with the intelligent wheelchair Sharioto of the KU Leuven [10] to allow a person to continuously drive it in natural environments, as shown in Figure 1. This brain-actuated wheelchair incorporates the advances in adaptive shared autonomy, on-line adaptation, as well as the on-line use of both high frequency bands and estimated local field potentials [11], four of the achievements of the MAIA project.

This paper presents this adaptive shared control system for the BCI controlled wheelchair where several behaviours are enabled simultaneously and will be activated only when the user is in need of them. In this paper, we will focus on these aspects that have been tested in simulation in order to evaluate the proposed approach. The use of shared control techniques has a profound impact on the performance during BCI control of a robotic assistant [12]. Yet, most of the time, shared control techniques assist the user in a constant and identical manner every time. In other words, the level of assistance is constant.

A next step in the development of shared control techniques would be to make the robot's assistance level adaptive so as to complement the user's capabilities at any moment. The better the user can do by himself, the less assistance he receives from the shared control system; and vice versa. In this way, the user remains in maximal control of the brainactuated robot, which is considered to be desirable for people in need of such systems [1]. To implement this principle, it is necessary for the shared control module to detect when and in what way the user needs assistance. An appropriate assisting behaviour would then be activated for the time that the user needs help. In other words, the system should be able to constantly adapt the level of assistance to the specific situation. More assistance when the user needs it, less when the user is sufficiently capable of controlling the robot himself.

In the experiment presented in this paper, we tested this concept of adaptive shared control by introducing three levels of assistance which are activated only when the user needs them. The first two, collision avoidance and obstacle avoidance, prevent the user from colliding with obstacles. The third level of assistance is called orientation recovery and will be triggered whenever the user has difficulties in driving the wheelchair towards the goal.

In this case, however, rather than choosing one of them, all behaviours are enabled, but they will only be active if their respective assisting behaviour is required. Therefore, the user has complete control over the wheelchair until he or she requires assistance. The goal of this experiment is to indicate the need for such an adaptive shared control as well as the benefits the user will gain from it. This paper

${ }^{1}$ MAIA or Mental Augmentation through Determination of Intended Action, is a EU STREP IST project (6th FWP). The coordination is done by IDIAP, Martigny, Switzerland and other partners are Katholieke Universitei Leuven (B), University Hospital of Geneva (CH), Fondazione Santa LuciaRome (I) and Helsinki University of Technology (F). More information can be found on http://www.maia-project.org focuses on the shared control framework, rather than on the BCI itself.

\section{APPROACH}

\section{A. Brain-Computer Interface}

The mental commands are obtained from a BCI based on non-invasive electroencephalogram (EEG) signals. These signals are measured by placing electrodes on the scalp, after preparing the scalp area by applying a conductive gel to reduce impedance. The electrodes are part of the EEG cap, worn by the subjects. The measured EEG signals represent an electrical signal (post-synaptic potentials) from a large number of neurons. An amplifier is connected to the electrodes on the EEG cap to amplify the voltage signal. The resulting signal is filtered by a high-pass filter and a low-pass filter. Finally, the signal is sent through an analogue to digital converter. EEG potentials were recorded at $512 \mathrm{~Hz}$ with 64 electrodes covering the whole scalp. An example of such a configuration is depicted in Figure 1.

There are three possible discrete mental steering commands: Forward, Left and Right. An asynchronous BCI, which responds every 0.5 seconds, sends a probability distribution over the three mental commands to the shared control system. This probability distribution is estimated by a statistical Gaussian classifier that takes as inputs samples made of the power spectrum density, computed over the last second, at several frequency bands for a number of channels. Frequency bands and channels were individually selected using feature selection techniques, yielding 4 bands (from $8-14 \mathrm{~Hz}$ to $192-208 \mathrm{~Hz}$ ) and 4 to 8 electrodes per experimental subject. Details of the BCI and the statistical classifier can be found in [8], [9].

\section{B. Shared control}

The estimated probability distribution is sent from the BCI system to the shared control system, which translates these probabilities to proper joystick-like input values, represented by a translational $(v)$ and rotational $(\omega)$ velocity. The steering command with the highest probability is considered the user's current steering intent and used further on as input. This steering command will result in the proper motion of the wheelchair. Issuing a Forward command, i.e. the probability of Forward was higher than the probability of Left and the probability of Right, results in an increase of the translational velocity $v$. Left and right steering commands represent the user's intent to rotate the wheelchair and decrease or increase the rotational velocity $\omega$, depending on the direction. After sending a Forward command, it is maintained for some time, to provide a smoother motion of the wheelchair and avoiding the need to reissue the same command every 0.5 seconds. If the user sends a Left or Right command during this time, the resulting joystick input will be a combination of an positive translational velocity and, depending on the direction, a positive or negative rotational velocity.

Instead of directly executing the user's steering commands, the shared control system evaluates the situation. The current environment, registered through a laser scanner, is taken into 


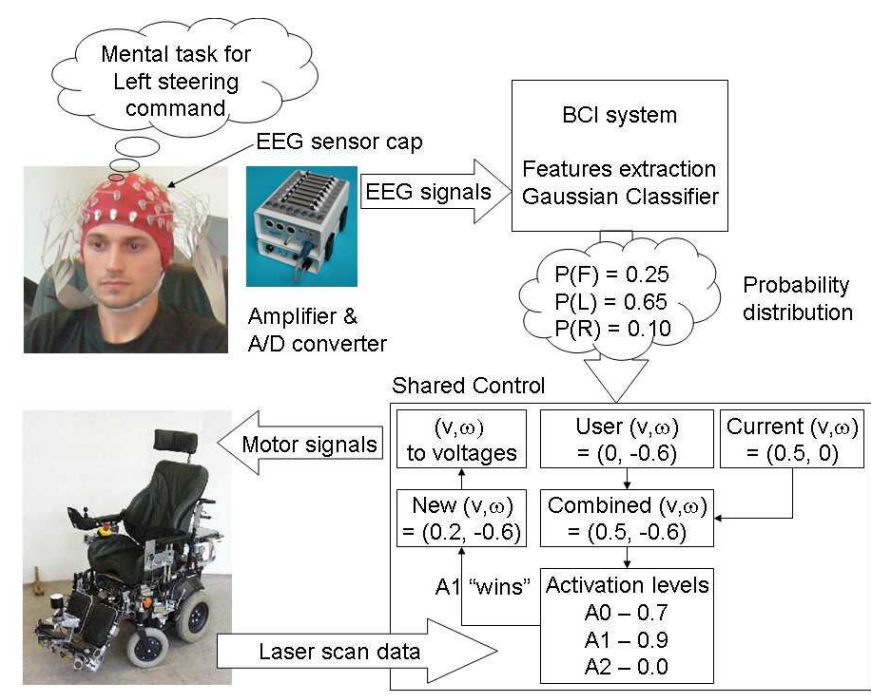

Fig. 2. Diagram of how a mental steering command is integrated in the shared control system and eventually converted into motor signals. Shown here, the user's mental task corresponds to moving the wheelchair left. In this case, obstacle avoidance is the winning behaviour and adjusts the $(v, \omega)$ velocities to prevent collision.

account. All assisting behaviours have an appropriateness level. Given the environmental information, each behaviour calculates its appropriateness. The shared control system then applies winner-takes-all to determine which behaviour it activates. The winner-takes-all algorithm activates the assisting behaviour with the highest appropriateness level. For example, if the user steers too close to an obstacle, an avoidance behaviour of the shared control system will generate a higher appropriateness level than that of the other behaviours and, thus, it will be activated be the shared control system in order to prevent collision.

A diagram of the translation from mental steering command to actual motor signals is shown in Figure 2. In this case, the actual steering command is adjusted by obstacle avoidance to prevent collision with nearby obstacles.

\section{Adaptive levels of assistance}

The shared control framework we propose here introduces three levels of assistance, named respectively $A 0, A 1$ and $A 2$, which are only activated when the user requires them. The first two, collision avoidance and obstacle avoidance, will be activated near obstacles to prevent collisions. A third level of assistance, called orientation recovery, will trigger whenever the user's direction is misaligned too much with respect to the goal direction.

1) AO - collision avoidance: The collision avoidance acts as an emergency stop. If the user steers the wheelchair too close to an obstacle, this behaviour decreases the translational velocity until the wheelchair comes to a full stop. The laser scanner, in front of the wheelchair, is used to determine the activation of this behaviour. The activation threshold in this experiment was set at $0.4 \mathrm{~m}$. If the system detects obstacles within this threshold, the appropriateness level of this behaviour is high, otherwise it is low.

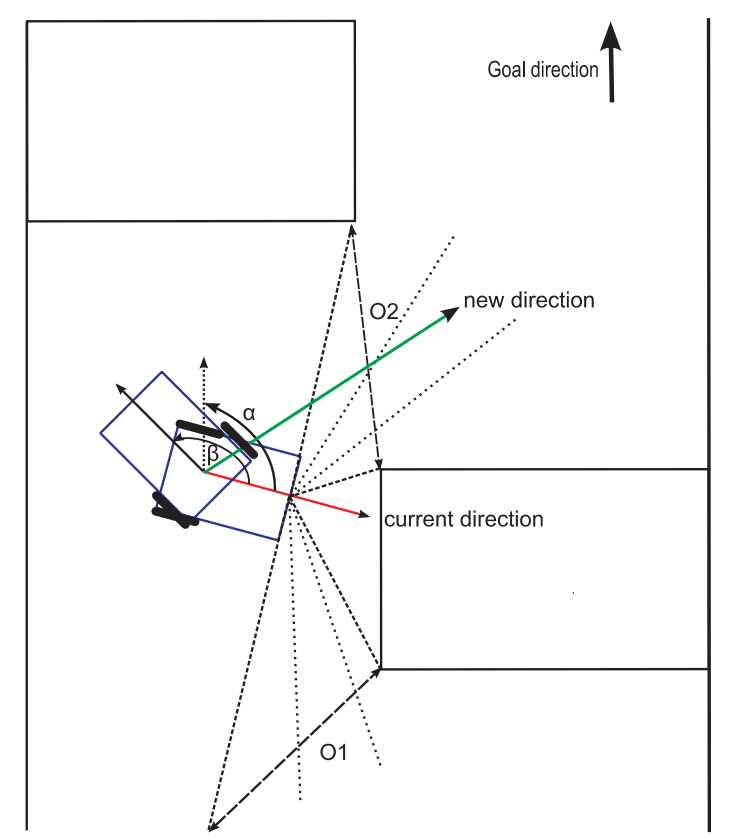

Fig. 3. The orientation recovery behaviour. If the angle between the current orientation of the wheelchair and the goal direction, given by $\alpha$, reaches a certain threshold, the behaviour will correct the orientation as follows: first, the wheelchair will be rotated over a certain angle, given by $\beta$, in order to scan for valid openings; secondly the opening with a direction closest to the goal direction is chosen; finally the wheelchair will center its direction towards the chosen opening direction. In this case the direction of opening $O 2$ is closest to the goal direction and is chosen, even though the larger opening more south, $O 1$, is a valid opening.

2) Al - obstacle avoidance: Unlike the previous behaviour, obstacle avoidance calculates a proper $(v, \omega)$ pair to steer the wheelchair away from the obstacle. During calculation the input of the user and the environment itself are taken into account, to assist appropriately. The activation threshold of this behaviour is set at $0.5 \mathrm{~m}$. Details of the both avoidance algorithms, A0 and A1, can be found in [13].

3) A2 - orientation recovery: The orientation recovery algorithm corrects the orientation of the wheelchair if it is too misaligned with respect to the goal orientation. Figure 3 illustrates this behaviour. The rectangle represents the wheelchair with its current orientation towards the south east. If in this case the user keeps turning right, the angle $\alpha$, which measures the angle between the current orientation of the wheelchair and the goal direction, will increase. Whenever $\alpha$ reaches a certain threshold, in this experiment set at 105 degrees, orientation recovery will be activated.

First, the direction, in which the wheelchair needs to turn to realign with the goal direction, is calculated. Depending on the current orientation of the robot and the goal orientation, the shortest path is chosen. In Figure 3, the desired direction is left. In the next step the algorithm calculates the best nearby opening. Best in this case is defined as having an orientation closest to the goal direction. A candidate opening is a set of consecutive distance measurements of a scan, which are larger than some threshold. If the width of the opening is larger than the width of the robot increased with a safety margin, the opening is considered as valid for the 


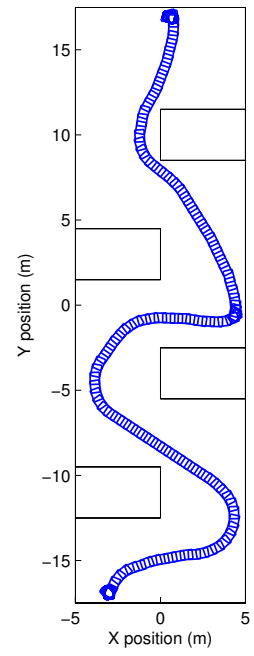

Fig. 4. The environment in which the experiment was performed. The trajectory of the wheelchair during the session is depicted as a sequence of squares.

robot to pass through safely. Because no global map is used, it is necessary to scan through the environment for openings in order to determine the best one. If a global map would be used, the best opening will be known at all times without needing to rotate the robot.

The algorithm rotates the wheelchair around its axis for a certain amount of degrees, given by the angle $\beta$. In this experiment $\beta$ was set at 150 degrees. At each step, the laser scanner will scan for 180 degrees in front of the robot and for each of the 180 measurements the distance to the closest obstacle is given. Using these scan data, openings are calculated. In Figure 3, $O 1$ and $O 2$ are considered valid openings for the first step. During the rotation all the other valid openings are also stored. Each valid opening has an orientation, relative to the wheelchair. Out of all the candidate openings, the one with an orientation closest to the goal direction is chosen. If no openings were found, the search radius is widened, by increasing the angle $\beta$.

Finally, the robot will be rotated towards the selected opening. The direction to turn towards is recalculated to ensure the shortest angle is chosen.

\section{EXPERIMENTS AND RESULTS}

\section{A. Setup}

Experiments were carried out in a simulated environment by two able-bodied voluntary subjects. In two half days, the two subjects were asked to control the simulated wheelchair and drive it through the environment from the starting position at the bottom to a goal region at the top as depicted in Figure 4. The sequence of squares represents the trajectory of the simulated wheelchair during one of such sessions. The position is given in meters on each axis. Each subject had one half of the day to perform several sessions. Subject 1 did 9 sessions in the morning, while subject 2 did 10 in the afternoon. Both subjects had already some limited experience with controlling the simulated wheelchair by BCI
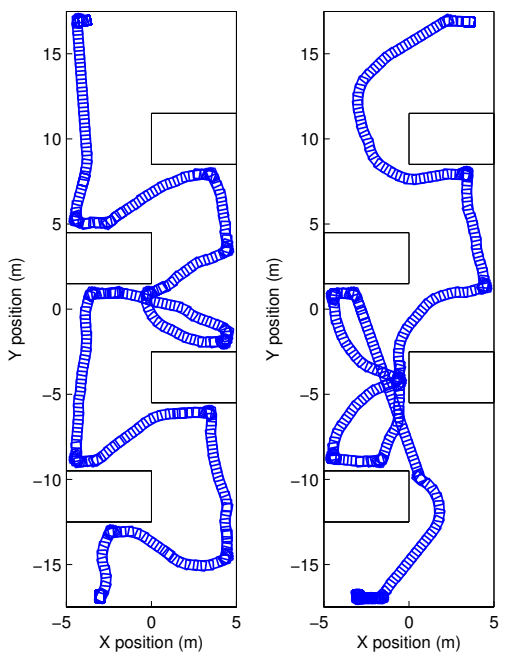

Fig. 5. The left trajectory is from a session of subject 1 and the right one is from a session of subject 2 . In both cases orientation recovery is disabled.

commands. Before each session, the level of assistance of the shared control system was set to either enable or disable the orientation recovery behaviour to establish the need for such a behaviour.

\section{B. Experiment}

The idea of the experiment was to test the need for the orientation recovery behaviour $(A 2)$, providing more assistance when the user needs it. During the whole session the two avoidance behaviours were enabled, to prevent collisions with any obstacles. Although they were enabled, the behaviours only actively intervened when the user moved too close to an obstacle. The need for the additional orientation recovery behaviour can be expressed in the difference in performance between sessions where $A 2$ was enabled and sessions where it was not. Performance criteria were distance of the trajectory and elapsed time to reach the goal region. Besides these two criteria, the BCI performance and the number of times $A 2$ is needed were compared to see if there might be any correlation.

\section{Results}

During the experiment, data were logged on different levels. First of all, the incoming mental commands, sent by the BCI classifier, together with the user's intent were saved. Also, the total time spent and total amount of distance travelled in each session was logged. The activation of the different levels of assistance was also stored, to be able to calculate the percentage of activation of orientation recovery in the sessions where it was enabled. Finally, the wheelchair's position and orientation were logged to make a plot of the trajectory, such as the one shown in Figure 4, and to calculate the need of $A 2$ in the sessions where it was disabled.

1) Distance: For both subject 1 and subject 2, the sessions where orientation recovery was enabled are executed with a lower total amount of travelled distance. Figure 5 shows that both subjects do make some loops when $A 2$ is disabled (7 

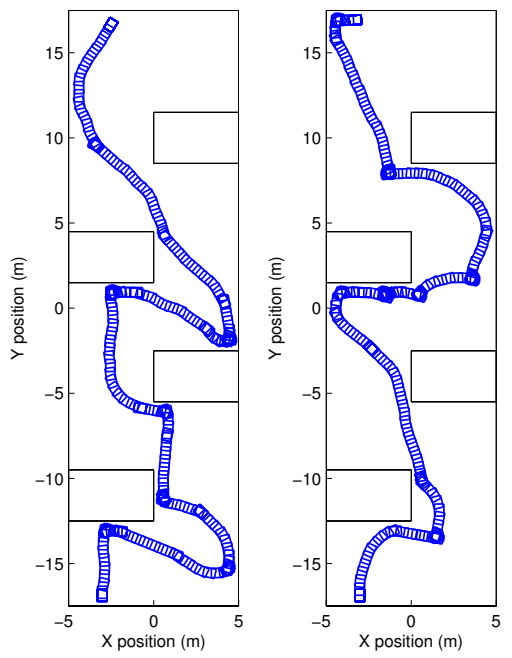

Fig. 6. At the left, orientation recovery is disabled and at the right it is enabled. Both trajectories are executed by subject 1 .

on 4 sessions and 6 on 6 sessions, respectively). Thus, orientation recovery could definitely help here. Figure 6 shows the difference in turns between sessions where orientation recovery is turned off and a session where it is turned on. Both trajectories were executed by subject 1 .

In Figure 7(a) the data on the distance is plotted in several box plots and below, in Figure 7(b), the averages are plotted with their respective standard deviations. For each subject, Figure 7 shows an overall statistic over all his or her sessions, a plot of the sessions without $A 2$ and a plot for the ones with A2 enabled.

First of all, it seems that subject 2 performed much better than subject 1 (with an average of $60.57 \mathrm{~m}$ versus $69.23 \mathrm{~m}$ for subject 1 ). This is explained by a better BCI performance, measured by the percentage of correctly classified steering commands, (58.10\% versus $53.69 \%$ for subject 1 ) as well as a better driving strategy: subject 1 took wider turns and switched slowly between BCI commands, while subject 2 took sharp turns and was able to switch faster.

Secondly, the standard deviation of the average distances between the different sessions where $A 2$ is turned on, and the interquartile distances in the box plots are also smaller than the ones between the sessions where it is turned off. When the user is doing really bad and no orientation recovery is turned on, many loops will occur and the distance will be much larger. However, if the agent is turned on, the performance of the user is more constant, because the agent reorients the wheelchair, whenever it is off course.

It is also noticeable that, even though subject 2 seems to have a better overall performance than subject 1 , he still performs better with the orientation recovery behaviour turned on, than without it.

If we divide all sessions into two groups, one group containing all sessions where $A 2$ is turned on ( 8 sessions) and the other group where it is turned off (11 sessions), a two-sample t-test confirms our claim that the distance travelled is significantly smaller when using $A 2$. We obtain a

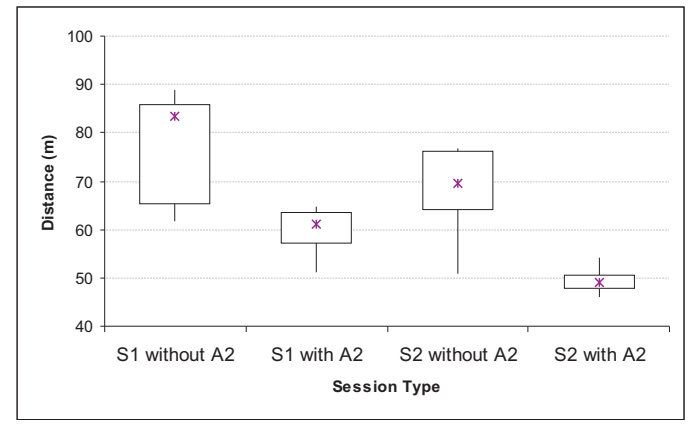

(a) Box plots

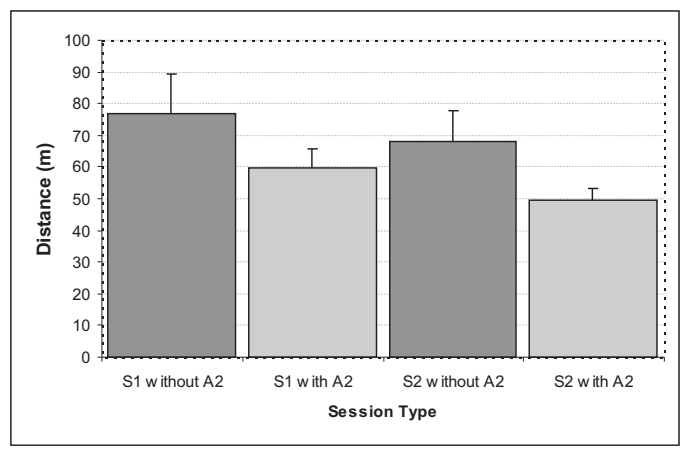

(b) Averages

Fig. 7. Box plots and averages of travelled distance with respect to different session types.

$95 \%$ confidence interval on the difference between the means of ] $-27.274 ;-7.643$ [ with an observed value $t=-3.753$. The $p$-value of 0.002 is lower than the significance level $\alpha=0.05$. Also the non-parametric two-sample Wilcoxon, Mann-Whitney test rejects the null hypothesis with a $p$-value of 0.002 at significance level $\alpha$ of 0.05 .

To conclude we could state that regarding distance travelled, $A 2$ is a benefit. Inexperienced users, like ours, will travel much less distance and the behaviour will be frequently active. Experienced users, although they might not need it all the time, can still rely on orientation recovery to help them in the few cases they do make a wrong turn (due to concentration problems or fatigue, for instance).

2) Time: The elapsed time is also reduced, on average, although the difference is smaller than the difference with respect to distance travelled (see Figure 8). This is due to the fact that the orientation recovery agent needs to scan the environment, looking for the most suitable opening to reorient the wheelchair towards. While $A 2$ scans through the local environment, by rotating the wheelchair, time is lost.

If we divide all sessions into two groups again, a twosample t-test as well as a Wilcoxon, Mann-Whitney test return a $p$-value higher than the significance level alpha= 0.05 ( 0.162 and 0.206 respectively), which indicates that the difference of elapsed time between the two groups is not significant. Nevertheless, on average, the elapsed time for sessions with $A 2$ turned on was smaller than those without assistance. 


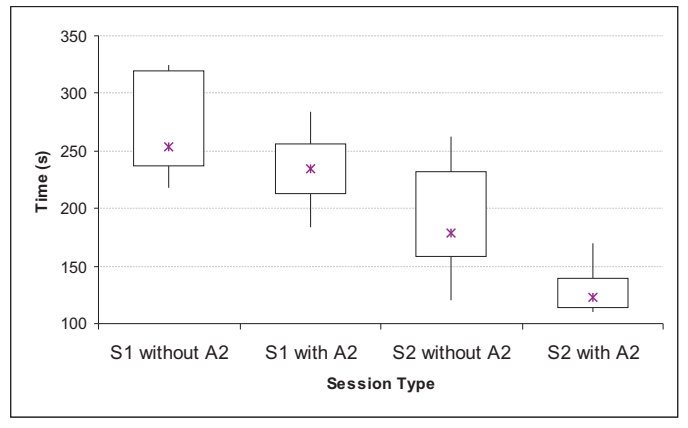

(a) Box plots

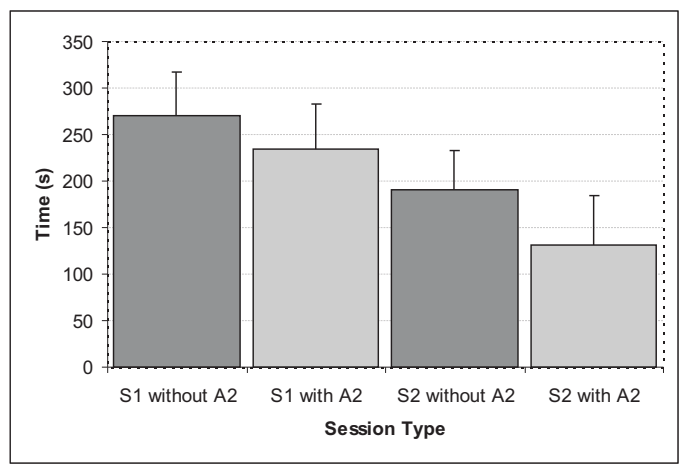

(b) Averages

Fig. 8. Box plots and averages of elapsed time with respect to different session types.

3) The need for A2: Also, if we compare, on average, the need for $A 2$ (i.e. the number of times $A 2$ could have helped by reorienting but was disabled or did help by reorienting when it was enabled) with the BCI performance for both subject 1 and subject 2 , we can see that with a higher BCI performance the number of times $A 2$ was active or could have been active is lower than with a lower BCI performance. This can be seen in Table I. A more experienced user has less need for $A 2$ than an inexperienced one.

4) BCI performance: The performance of the BCI is rather low for the two experimental subjects, although significantly better than random (33.3\% for a 3-class problem). One of the reasons for this low performance, despite their previous limited experience in controlling mentally the simulated wheelchair, is that, after the introduction of $A 2$, the wheelchair has a different behaviour and the subjects need to learn its new dynamics.

Nonetheless, it is remarkable that a BCI performance of around $55 \%$ is sufficient to drive the wheelchair along the corridor. The main reason is that this performance corresponds to the correct mental command issued every half a second, and not the achievement of subtasks such as move towards the next passage, negotiate obstacle to the right, or turn left to cross the passage. To achieve these subtasks the wheelchair needs basically to move forward, turn right, and turn left. Thus, for instance, the wheelchair can progress towards the next passage even if it turns left or
TABLE I

AVERAGE BCI PERFORMANCE OF BOTH SUBJECTS COMPARED WITH THE AVERAGE NUMBER OF TIMES THEY NEEDED $A 2$

\begin{tabular}{|c|c|c|}
\hline Subject & BCI Performance & $\# A 2$ needed \\
\hline 1 & $53.69 \%$ & 5 \\
\hline 2 & $58.10 \%$ & 1.7 \\
\hline
\end{tabular}

right occasionally. And this example brings forth the second reason why a low instantaneous BCI performance suffices for driving the wheelchair: incorrect mental commands do not hinder progress provided a large number of correct commands are issued at critical moments.

\section{CONCLUSIONS AND FUTURE WORK}

We have shown that the use of an adaptive level of assistance increases the task performance. The assisting behaviours will only be activated when the user requires assistance and depending on the situation, the proper behaviour is activated. By introducing this adaptivity, the users remain in maximal control. An inexperienced user will receive more assistance than an experienced one. If, after some time, the performance of the user has improved, the assisting behaviours will be activated less.

In the experiment we showed the travelled distance and elapsed time of a session decreased while orientation recovery was active, resulting in an increase of the task performance. Also the need for this behaviour was given. It can be beneficial for both inexperienced and experienced users. The former will have a much shorter trajectory due to a frequently active $A 2$ behaviour and the latter, although it might not always be required, can still rely on orientation recovery. In the few cases they do make a wrong turn, the $A 2$ behaviour can also assist them.

A weak point of the proposed approach are the fixed activation levels, which do not integrate the user's experience or performance. The behaviour will always be activated when the activation threshold is reached, even though an experienced user might still be able to recover from this disorientation on his own.

We could increase the performance if we could build a model of the user at runtime and estimate the level of experience to determine the thresholds when the behaviour should be activated or not.

\section{REFERENCES}

[1] M. Nuttin, E. Demeester, D. Vanhooydonck, and H. Van Brussel, "Obstacle avoidance and shared autonomy for electric wheel chairs: An evaluation of preliminary experiments," in Robotik 2002, Ludwigsburg, Germany, June 2002.

[2] D. Vanhooydonck, E. Demeester, M. Nuttin, and H. Van Brussel, "Shared control for intelligent wheelchairs: an implicit estimation of the user intention," in ASER, Bardolino, Italy, March 2003, pp. 176182.

[3] E. Demeester, M. Nuttin, D. Vanhooydonck, and V. B. H., "A modelbased, probalistic framework for plan recognition in shared wheelchair control: Experiments and evaluation," in IEEE/RSJ International Conference on Intelligent Robots and Systems (IROS), Las Vegas, Nevada, October 2003, pp. 1456-1461. 
[4] M. Betke, J. Gips, and P. Fleming, "The camera mouse: Visual tracking of body features to provide computer access for people with severe disabilities," IEEE Transactions on Neural Systems and Rehabilitation Engineering, vol. 10, no. 1, pp. 1-10, 2002.

[5] A. Kübler, B. Kotchoubey, J. Kaiser, and N. Wolpaw, J. Birbaumer "Brain-computer communication: Unlocking the locked in," Psychological Bulletin, vol. 127, no. 3, pp. 358-375, 2001.

[6] J. Wolpaw, N. Birhaumer, D. J. McFarland, G. Pfurtscheller, and T. M. Vaughan, "Brain-computer interfaces for communication and control," Clinical Neurophysiology, vol. 113, no. 6, pp. 767-791, 2002.

[7] J. del R. Millán, "Adaptive brain interfaces," Communications of the ACM, vol. 46, pp. 74-80, 2003

[8] J. del R. Millán, F. Renkens, J. Mouriño, and W. Gerstner, "Noninvasive brain-actuated control of a mobile robot by human EEG," IEEE Trans Biomed Eng, vol. 51, pp. 1026-1033, 2004.

[9] J. del R. Millán, P. W. Ferrez, and A. Buttfield, The IDIAP Brain Computer Interface: An Asynchronous Multi-Class Approach, in G. Dornhege et al. (eds.), Towards Brain-Computer Interfacing. MIT
Press, 2007.

[10] K. U. Leuven, "Mobile learning robots research group (mlr)," URL, Februari 2007. [Online]. Available: http://www.mech.kuleuven.be/mlr/

[11] P. W. Ferrez, F. Galán, A. Buttfield, S. L. Gonzalez, R. Grave de Peralta, and J. del R. Millán, "High frequency bands and estimated local field potentials to improve single-trial classification of electroencephalographic signals," in Proceedings of the 3rd International BrainComputer Interface Workshop \& Training Course 2006, Graz, 2006.

[12] E. Lew, M. Nuttin, P. Ferrez, A. Degeest, A. Buttfield, G. Vanacker, and J. del R. Millán, "Non-invasive brain computer interface for mental control of a simulated wheelchair," in Proceedings of the 3rd International Brain-Computer Interface Workshop \& Training Course 2006, Graz, 2006

[13] M. Nuttin, D. Vanhooydonck, E. Demeester, and H. Van Brussel, "Selection of suitable human-robot interaction techniques for intelligent wheelchairs," in Proceedings of 11th IEEE International Workshop on Robot and Human Interactive Communication ROMAN, Berlin, September 2002, pp. 146-151. 